\title{
Matched Tissues
}

National Cancer Institute

\section{Source}

National Cancer Institute. Matched Tissues. NCI Thesaurus. Code C92469.

Tissues samples from a single source that differ minimally (e.g., normal and cancerous tissues, treated and untreated tissues). 